$\xi=1$ 罥

\title{
E-mastering application utilizing pertinence heuristics evaluation for children
}

\author{
D. Lakshmi ${ }^{1 *}$, R. Ponnusamy ${ }^{2}$ \\ ${ }^{1}$ Research Scholar, Department of Computer Science and Engineering Sathyabama University, Chennai, India \\ ${ }^{2}$ Professor, Department of Computer Science and Engineering, Sri Lakshmi Ammal Engineering College, Chennai, India \\ *Corresponding author E-mail: lkdlak@gmail.com
}

\begin{abstract}
Choosing ease of use assessment techniques (UEMs) to uncover convenience issues in e-learning projects is affected by time, cost, simplicity of use, and effectiveness. Heuristic assessment has turned into a generally acknowledged technique for ease of use assessment in programming improvement. This paper presents Heuristic Assessment for Tyke E-learning applications (HECE), an exhaustive arrangement of heuristics for kid e-learning alongside a definite clarification for the ease of use specialists on the most proficient method to apply them. These arrangements of heuristics depend on Nielsen's unique ten heuristics produced for programming. Nielsen heuristics are essentially nonexclusive, and won't not include ease of use credits particular to kids or e-learning. The new HECE set would conquer these weaknesses. The legitimacy and viability of these heuristics were assessed against two created e-learning programs composed by ReDSOFT for KG-2 and exceptional need understudies. The outcomes showed that HECE distinguished subjective likenesses and contrasts with client testing, and that HECE is most appropriate for assessing general and kid ease of use. Consolidated with client testing, HECE offers another track that can help with directing the kid e-learning industry to outline applications that are both instructive and pleasurable for youngsters.
\end{abstract}

Keywords: E-Mastering; Human Computer Interaction; Heuristics Evaluation.

\section{Introduction}

These days e-learning applications is viewed as a quickly developing field in the product outline industry. As indicated by conventional advertising insight, the ease of use of an application must be examined accurately to make fruitful items. In the e-learning industry the test is to plan e-learning applications that are both instructive and charming for the learners. Assessing the convenience of e-learning applications represents its own necessities; henceforth, its ease of use assessment is unique in relation to that of general undertaking focused frameworks and requires distinctive criteria. This is a developing territory in which inquire about has been led by, among others, [1], [2], [3], [4]. The assessment of elearning ought to address parts of instructional method and gaining from instructive spaces, and also ease of use variables, for example, the productivity, viability and fulfillment of interfaces.

Heuristic assessment is a procedure for exploring the ease of use of programming initially created by Nielsen and Molich, and later refined by Nielsen [5], [6]. Advanced for

its cost proficiency and simplicity of usage, the HE technique comprises of at least one encountered evaluators (3-5 suggested) applying a built up set of rules called heuristics as they survey a given application. It is likewise called the "assessment" technique or 'markdown strategy' of ease of use assessment. HE is viewed as a conservative other option to trial convenience tests including real clients. HE has likewise been accounted for to be the most utilized convenience assessment technique [6]. The conventional heuristics detailed by Nielsen are fundamentally bland, and won't not envelop ease of use ascribes particular to kids or e-learning. Subsequently, there is a need to build up a comparing set of heu- ristics that consider the particular needs of kids and additionally the necessities of e-learning applications. This paper intends to investigate and propose another arrangement of heuristics to assess youngster e-learning applications.

The utilization of influential procedures and conduct adjustment systems for the plan of innovations that advance solid practices has been a noteworthy research pattern of the previous decade [1]. Specialists and industry both have built up various applications that utilization programming, portable innovations, diversions, and sites that urge individuals to exercise more [5], eat all the more steadily [4], rest better and quit smoking. One of the difficulties in planning for this space is in assessing the viability of these advancements, particularly along the more powerful viewpoints. Standard UI assessment methods might have the capacity to address a portion of the convenience angles, however are generally poor apparatuses for surveying how fruitful an innovation might be in advancing conduct change or the probability of long haul selection. Along these lines, better approaches for assessing convincing advancements for wellbeing have been a current concentration of various specialists in this space [9], [10].

Heuristic assessment has been a work of art and exceptionally well known assessment strategy in the human-PC communication group as far back as it was first presented by Jakob Nielsen in 1990. This markdown ease of use procedure empowers a couple of master evaluators to survey an innovation curio for issues that may influence its ease of use in a short measure of time along an arrangement of rules known as heuristics.

Experts have revealed that heuristic assessment is a decent approach to discover huge ease of use issues at many phases of the outline procedure. Nielsen's strategy has been broadly received, and as of late, there has been a pattern to create and approve more 
particular heuristics for more specific advances. The expectation of the specific heuristics is to consider assessment of issues past ease of use and to concentrate evaluators on the themes that matter most to innovation originators and the plan of the innovation. Others have adjusted heuristics for advancements, for example, encompassing presentations, collective advances, robots, and computer games [4] and have been fruitful in distinguishing issues particular to those advances, and at times, more serious issues than the customary heuristics Nielsen depicts [9].

Whatever is left of this paper is composed as takes after. We initially give a depiction of the related work in the zones of influential wellbeing advancements, assessment of convincing advances, and heuristic assessment. We next present the 10 new heuristics we created alongside a depiction of how they were resolved. Taking after the heuristics, we portray the review we intended to contrast the new heuristics with Nielsen's unique 10 and afterward depict the outcomes. We talk about the discoveries and complete the paper.

\section{Related work}

Heuristics Assessment (HE) is a casual strategy where master evaluators logically look at the ease of use related parts of a UI [6]. HE is more subjective than customary client testing assessment since it is vigorously needy upon

the evaluators' abilities [7].The principle favorable position of HE is its cost-viability; it doesn't require refined research center hardware to record clients collaborations, costly field examinations, or difficult to-process comes about because of across the board meets [6]. The evaluator alone can recognize an extensive variety of ease of use issues and conceivable shortcomings of a perplexing framework in a constrained measure of time [6], [7]. Hence, HE has accomplished across the board use as of late, particularly in mechanical situations [9].

\subsection{Persuasive technologies for health}

In the previous two decades, powerful advances have entered the commercial center at an undeniably quickened rate. Albeit enticing advancements fill various needs, some contend that their most critical commitment is in the space of wellbeing. Enticing advances for wellbeing as of now traverse an assortment of use ranges [8]. A portion of the more famous ones incorporate advancing physical wellness. Administration of ceaseless infection is another territory where PDAs and sites are extending our abilities. There has even been some work in advancing individual cleanliness, for example, tooth brushing for kids [2]. This rundown of utilization zones and influential advances for wellbeing is in no way, shape or form far reaching however gives an understanding into their immense area. The exploration we directed expected to take into consideration the simpler assessment of advances from any wellbeing space.

In spite of the fact that sites may right now be the most widely recognized stage for influential advances, this won't be the situation sooner rather than later. Truth be told, wellbeing related convincing innovations are as of now being created for an assortment of stages including mobile phones [5],[6], interpersonal interaction sites 7,8, and computer games. In this work, we have made a technique for assessing the viability of powerful advances paying little respect to their frame figure, application sort, or stage.

\subsection{Evaluation of persuasive technologies}

Enticing innovations can be extremely hard to assess for a few reasons. The principle reason concerns the way that measuring the adequacy of enticing advancements requires a one of a kind comprehension of both the persuader and persuadee, notwithstanding how they communicate with each other [3]. Therefore of this multifaceted nature, numerous convincing wellbeing innovations are assessed through reviews that require huge spending plans and a considerable measure of exertion notwithstanding using completely useful models. These sorts of costly reviews are not generally an alternative and in some cases ignore long haul powerful impacts. For this and different reasons, there is a requirement for the further advancement of assessment procedures that can rapidly and reasonably identify ease of use issues at an opportune time in the outline procedure of enticing advances for wellbeing, for example, a particular heuristic assessment. Heuristic assessment is a minimal effort and open choice that is as of now one of the top systems presently utilized by ease of use specialists [6]. Hence, we are trying to enhance the assessment techniques for use by architects of influential innovations for wellbeing, which might be utilized as a part of deciding their ease of use and probability of selection, achievement, and influence.

\subsection{Heuristic evaluation}

Heuristic assessment is a casual technique for ease of use testing that comprises of various evaluators that are given an interface plan, and after that are made a request to remark on the mistakes and adequacy of the item. What's more, heuristic assessments are a minimal effort, quick, and proficient strategy for having the capacity to distinguish any ease of use issues that may happen with the item. Having individuals direct these assessments as per certain tenets is the perfect strategy for utilizing heuristic assessment. A great many people who utilize heuristic assessments would perform them in view of their instinct and judgment skills. As of now, there are 10 suggested general ease of use heuristics that depend on Nielsen's strategies for heuristic assessment:

Deceivability of framework status

- Coordinate amongst framework and this present reality User control and opportunity

- Consistency and norms

- Mistake anticipation

- Acknowledgment instead of review

- Adaptability and effectiveness of utilization

- Tasteful and moderate outline

- Enable clients to perceive, analyze, and recuperate from mistakes

- Help and documentation

Heuristic assessments can be connected to any intuitive mechanical item. Many research considers have utilized heuristic assessment on particular advances, for example, encompassing showcases, computer games, community oriented advances, and electronic therapeutic records, as we portray underneath. Most of the accompanying examination considers assessed an arrangement of new heuristics that they made and contrasted it with Nielsen's unique arrangement of heuristics, which filled in as a model for our review plan. All the more firmly identified with the domain of wellbeing innovations, heuristic assessment can be utilized to assess individual wellbeing record frameworks. The first heuristics and an adjusted form of heuristics were utilized on three individual wellbeing record frameworks [1]. The outcomes showed that the first 10 heuristics discovered numerous convenience mistakes; in any case, the adjusted variant of the heuristics performed better with a similar number of evaluators. Our work varies in that we concentrate basically on advances intended to convince the end client to be more advantageous, while individual wellbeing records are principally for capacity and access to medicinal records[3]. In general, heuristic assessment is a gainful technique that can help recognize disparities in any item. Be that as it may, certain outcomes may fluctuate contingent upon the item when utilizing the first arrangement of heuristic assessments, so it may be important to make an altered rendition of heuristics to uncover more issues. In this work, we look to reproduce a considerable lot of these past reviews yet utilize another area which has not yet been investigated. 


\section{Heuristics}

The initial phase in this exploration was to decide an arrangement of heuristics that we accept would better survey enticing advances intended to advance great wellbeing. In this area, we portray the procedure for characterizing and choosing the heuristics and afterward give the rundown of our 10 influential wellbeing heuristics and their definitions.

\subsection{Handle for defining heuristics}

Our approach was to survey the writing and gather an ace rundown of all ease of use rules and heuristics we could discover identifying with enticing advances. A gathering of 13 scientists and understudies with involvement in client focused outline and ease of use (counting the co-creators) each produced a rundown of 10 of what they accepted were the most essential rules and proposals found from the writing and from their own particular encounters utilizing and planning convincing innovations. A sum of 130 rules was produced. From this rundown, the analysts as a cofound gathering attempted to limit the rundown by joining the comparable rules, organizing them, and examining them utilizing a procedure like liking graphing. A few of the more particular rules were joined into one broader rule, with the specifics incorporated into the more distinct content. Altogether,

we limited the rundown of heuristics to 10 to keep a straightforward rundown that would not overpower evaluators and enable them to concentrate on the most essential viewpoints. This likewise enabled us to do a correlation with Nielsen's unique 10 . We take note of that there is some cover with Nielsen's unique rundown, and this is purposeful in light of the fact that by and by, this rundown would likely be utilized as a substitution for Nielsen's rundown and we didn't need evaluators to disregard assessing a portion of the more key ease of use standards.

\subsection{Persuasive health technology heuristics}

The following are the 10 last heuristics from the procedure we depicted. We gave every heuristic a short name and a more extended definition.

1) Appropriate Functionality: The innovation ought to meet ease of use, portability, deceivability, and toughness needs as indicated by the settings in which it may be utilized. The innovation ought to work viably in the client's condition by being anything but difficult to utilize and incorporate into one's day by day life and schedule.

2) Not Irritating or Embarrassing: The innovation ought not chafe or humiliate the client, even subsequent to utilizing the item more than once and routinely over a drawn out stretch of time. This identifies with viewpoints, for example, the nearness of the item itself in the client's condition, how much the innovation encroaches upon the client's day by day life, the planning, sort, exactness, and measure of criticism given, and the capacity for tweaked settings and protection controls.

3) Protect Users' Privacy: The framework enables clients to keep individual data private. Clients can control what, when, to whom, how, and how much data is made open. Any open data is kept conceptual.

4) Use of Positive Motivation Strategies: The innovation perceives when target practices have been performed or objectives have been met and uses encouraging feedback techniques to advance proceeded with advance. Maintains a strategic distance from utilization of discipline for inability to perform target practices or meet objectives.

5) Usable and Esthetically Appealing Design: The visual outline of the innovation is alluring and engaging and clings to essential convenience guidelines. The outline catches and supports the client's advantage, improves client engagement with the innovation, and furthermore adds to the believability and convenience of the item.

6) Accuracy of Information: The innovation ought not mistakenly record or distort the client's conduct (for example, because of confinements in programmed detecting capacities or the failure to utilize the gadget in specific conditions). On the off chance that vital - to get a precise, far reaching record of conduct - the innovation ought to enable clients to alter information records as well as physically info extra information that the gadget is unequipped for recognizing consequently. ics have been created to address some specific issues identified with UI plans.

Another arrangement of heuristics have been produced for playability by Desurvire et al. Their heuristics are worried with diversion play, amusement story, diversion component, and diversion ease of use. They had thought about the issues uncovered by their heuristics with those by client testing. Their outcomes showed that in spite of the fact that client testing uncovered issues that lone can be found by trying different things with genuine clients, playability heuristics uncovered more issues and there was a cover between the two arrangements of issues. An embeddings prescient assessment technique for ease of use and fun is Organized Master Evalaution Strategy (Appear to be produced by Baauw et al[5]. Appear's contain an agenda that comprise of inquiries in light of Norman's hypothesis of activity and fun related ideas from Molone. Appear's outcome are extremely encouraging in foreseeing convenience issues. Korhonen and Koivisto have built up an arrangement of heuristics to address the playability issue for versatile recreations from three perspectives, two identified with amusement interfaces and one identified with portable structure.

\section{Extended heuristics assessment}

This area portrays the two convincing wellbeing innovation items that we assessed with heuristic assessment. Both items are intended to advance conduct change in their clients [11]. As a gathering, we made a rundown of 13 promptly accessible enticing wellbeing applications that we could possibly assess. We at that point talked about the advantages of including each of the applications in our review and chosen to pick two advances that could be effortlessly circulated to remote ease of use analyzers and secured two diverse powerful methodologies, and in addition spaces and level of specificity. At last, we picked Mindbloom, an electronic application intended to track advance on your whole life's objectives, including wellbeing, wellness, social connections, and fund, and MyPyramid BlastOff! an online diversion to show kids about sound nourishment decisions. We take note of that the particular selection of advancements is not essential to the assessment of our heuristics other than giving agent cases of enticing innovations that secured an expansive range, and this review likely could have been imitated utilizing diverse advances.

\subsection{Mindbloom}

Mindbloom is an online application that enables clients to set short and long haul life objectives and needs and plans to assemble significant connections between clients. Mindbloom's essential clients are holistic mentors and their customers. A client's life is spoken to by an "Existence Tree" whose branches speak to life ranges critical to every individual client, e.g. wellbeing, most profound sense of being, connections, recreation, way of life, accounts, innovativeness, and profession. On a branch, each leaf speaks to an objective or dream identified with that life region. Applying the conventional heuristics defined by Nielsen in the assessments of tyke e-learning projects is very not attainable. In the first place, the current heuristics does not manage youngster prerequisites or e-learning angles, which are two noteworthy viewpoints in tyke e-learning applications. Second, Nielsen heuristics are not portrayed in detail with the goal that they could have been specifically adjusted to the kid e-learning improvement. 
Third, the unwavering quality of the outcomes is frequently altogether reliant on the individual know-how, mastery and abilities of the evaluator. This issue was likewise brought up by different specialists, who have characterized more particular rules for specific framework classes. Consequently, we expected to grow Nielsen's conventional ten heuristics to twenty-one heuristics with nitty gritty clarifications, to be all the more firmly centered around youngster e-learning applications and defeat the above inadequacies.Initially, we needed to characterize what sort of perspectives ought to be assessed. The general ease of use of kid e-learning projects is a critical perspective, as youngsters would prefer not to battle with an application, since they are not inspired by the UI. It ought to likewise consider the mental and physical capacity of the tyke. Consequently, the UI of the application ought to be exceptionally regular and instinctive to be utilized for instance by 6 to 10 years of age tyke. Since we are assessing e-learning applications, another perspective is to assess the academic adequacy. Moreover, the application needs to bolster learners in taking in the substance material in a manner that they commit least push to genuine association with the program, and it must make adapting more powerful and energizing.

\subsection{Mypyramid blast off}

MyPyramid Blast Off is a diversion intended to instruct young sters about the significance of adhering to a good diet and physical action. By exhibiting how youngsters can choose solid sustenances for their own eating regimens, the diversion convinces players to settle on savvy decisions about eating and exercise in their own particular lives. The amusement reenacts a mission to space in which players must fuel their rocket ship and charge their battery keeping in mind the end goal to achieve Planet Power. Players are told that the rocket requires one day of fuel, spoken to by nourishment, and a hour of movement to dispatch.

\section{Verification of the child e-learning}

The point of this exploration was to build up an arrangement of checked heuristics for kid e-learning applications. The main form of the Heuristic Assessment Youngster E-learning applications (HECE) depended on adjusting and arranging existing rules or relevant agendas into three classifications. A nitty gritty depiction of every heuristics has been added to help convenience evaluator specifically apply the heuristics while assessing the e-inclining program. HECE was surveyed by ease of use specialists and kid etaking in originators from Kuwait College and ReDSOFT. What's more, the created set of HECE was relatively tried against customary end - client testing. The outcomes were inspected to assess the legitimacy of the created set of heuristics, and to distinguish the qualities, shortcomings, and subjective contrasts of strategies based on Visibility of System Status, Match Between System and the Real World, User Control and Freedom, Consistency and Standards, Error Prevention, Recognition Rather than Recall, Flexibility and Efficiency of Use, Esthetic and Minimalist Design, Help Users Recognize, Diagnose, and Recover from Errors.

\section{Child usability heuristics}

With a particular true objective to endorse the HECE set of heuristics, heuristics appraisal and usability testing were driven and the results were penniless down and broke down. Two e-learning applications formed by ReDSOFT were surveyed and examined for their convenience; an e-slanting application for KG-2 and an almost deaf e-learning model; see Fig. 1 and Fig. 2. The e-learning application for KG-2 was proposed for five to six years old adolescent to learn essential scrutinizing, forming, math, and science capacities. Eight activities were surveyed by both convenience authorities and usability testing with adolescents. Advance, a nearly deaf e-learning model was expected for seven to nine years old children to learn new tones', numbers', animals' definitions, maps, and to scrutinize stories. Most of the five activities was considered in the appraisal methodology by the convenience authorities and usability testing with almost deaf children.

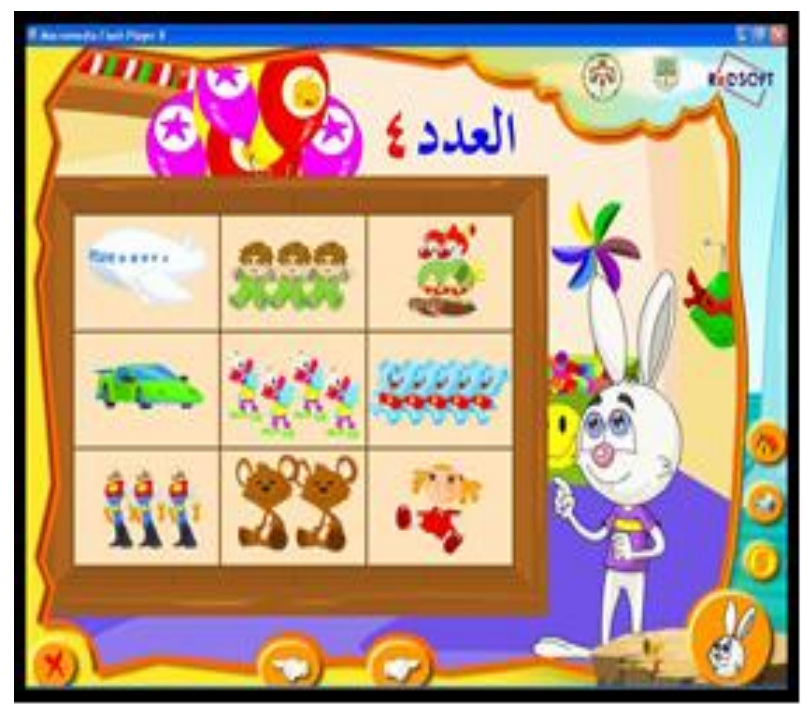

Fig. 1: User Interface Sample of Math Lesson for KG-2.

Heuristic Evaluation

Comfort pros from Kuwait University, ReDSOFT and e-taking in inclination from the Ministry of Education have appreciated the evaluation of the two e-learning programs using the HECE set. The evaluators focused on how every heuristic was satisfied or neglected, and a while later portrayed the tyke e-learning issues. For each predicated issue the evaluators expected to clear up the reality of the issue and suggest elective responses for settling the issues if possible.

For the KG2-e-getting the hang of programming, nine out of the ten Nielsen heuristics, five out of the six child usability heuristics, and three out of the five e-learning usability heuristics were useful in uncovering comfort issues. This reveals for kid e-learning design, HECE is important in every one of the three orders. Eight activities in the e-learning application were evaluated. Propel evaluation of the activities could find additional usability issues, which would give extra affirmation of the handiness of the HECE set. A huge part of the convenience issues were found in the Nielsen usability (29 issues) and the Child usability (24 issues) classes, while only seven issues were found in the E-learning grouping. This could be elucidated as takes in the wake of: demonstrating teachers from the Ministry of preparing and e-learning originator authority participated in working up this item. In any case, no usability ace partook in the headway.

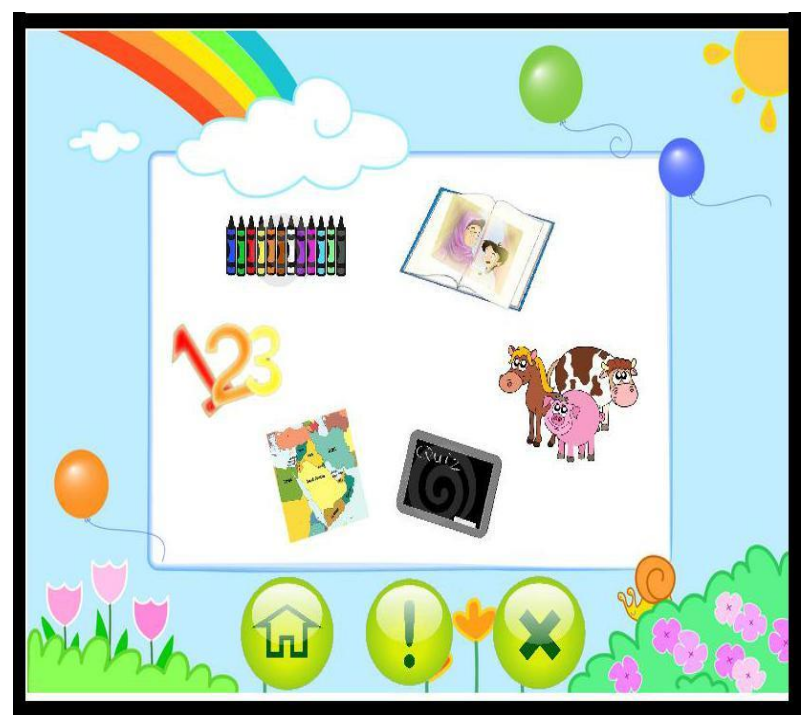

Fig. 2: User Interface Sample of Deaf E-Learning Prototype. 


\section{Conclusion}

This paper displays the Heuristic Evaluation for Child E-learning applications (HECE) set to play out a canny usability appraisal of adolescent e-learning applications. HECE was seen to be to a great degree helpful in evaluating adolescent e-learning applications, and to be most suitable to finding accommodation and e-learning issues appropriate on time in the change method. E-taking in applications may benefit by such a course of action of heuristics, since they give correct contribution to respects to adolescent elearning issues to the originators. Regardless, customer testing must at display be coordinated, as youth lead can be uncommon and specific issues must be revealed in the midst of such testing. Urge refinements to the HECE set are most likely going to be made in light of the limit of the heuristics to various youth elearning applications. Moreover work should in like manner focus on broadening the endorsement of the HECE set so usability evaluators can use it with assurance while surveying the layout of child e-learning application.

\section{References}

[1] M. A.Storey, B. Phillips, M. Maczewski, and M. Wang. "Evaluating the usability of Web-Based Learning Tools," Education Technology \& Society, vol. 5, pp.91-100, July 2002.

[2] B. Wong, T. T. Nguyen, E. Chang, E., and N. Jayaratna. "Usability Metrics for E-Learning," Workshop on Human Computer Interface for Semantic Web and Web Applications, Springer-Verlag, Heidelberg, Germany, LNCS no. 2889, pp. 235 - 252, 2003.

[3] C. Ardito, M. F. Costabile, M. De Marsico, R.Lanzilotti, Levialdi S., Roselli T., and V. Rossano, "An Approach to Usability Evaluation of e-Learning Applications," Universal Access in the Information Society International Journal, vol.4, no. 3, pp. 270-283, 2006. https://doi.org/10.1007/s10209-005-0008-6.

[4] A. De Angeli, M. Matera, M. F. Costabile, F. Garzotto, and P. Paolini, "On the Advantages of a Systematic Inspection for Evaluating Hypermedia Usability," International Journal of Human-Computer Interaction, Lawrence Erlbaum Associates, vol. 15, no. 3, pp. 315 335, 2003. https://doi.org/10.1207/S15327590IJHC1503_01.

[5] R. Molich, and J. Nielsen, "Improving a human-computer dialogue," Communications of the ACM, vol. 33, no.3, 338-348, March, 1990. https://doi.org/10.1145/77481.77486.

[6] J. Nielsen, Heuristic evaluation. In J. Nielsen \& R. L. Mack (eds.), Usability inspection methods, pp. 25-62, New York: John Wiley \& Sons, 1994.

[7] M. Matera, M.F. Costabile, F. Garzotto, and P. Paolini, "SUE Inspection: An Effective Method for Systematic Usability Evaluation of Hypermedia,” IEEE Transaction, vol.32, no. 1, January 2002.

[8] R. Jeffries, J. Miller, C. Wharton C., and K. M. Uyeda, "User interface evaluation in the real world: A comparison of four techniques," in Proc. ACM CHI, pp.119-124, 1991.

[9] K. H. Madsen, "Special issue on the diversity of usability practices," Communication ACM, vol.42, no.5, 1999. https://doi.org/10.1145/301353.301415.

[10] K.Sathesh Kumar, K.Shankar, M. Ilayaraja and M. Rajesh, "Sensitive Data Security In Cloud Computing Aid Of Different Encryption Techniques, Journal of Advanced Research in Dynamical and Control Systems, vol.18, no.23, 2017.

[11] K. Vijayakumar C,Arun, Analysis and selection of risk assessment frameworks for cloud based enterprise applications", Biomedical Research, ISSN: 0976-1683 (Electronic), January 2017. 